\title{
Distúrbios Psíquicos menores em trabalhadores que atuam na Estratégia de Saúde da Família: revisão integrativa
}

\section{$\underline{\text { Roan da Silva Gomes Sampaio }}{ }^{1}$; Rosely Cabral de Carvalho ${ }^{2}$; Gabriel Silva Rocha ${ }^{3}$;}

1. Bolsista PIBIC/FAPESB, Graduando em Medicina, Universidade Estadual de Feira de Santana, e-mail: roanrsgsampaio@gmail.com

2. Orientador, Departamento de Saúde, Universidade Estadual de Feira de Santana, e-mail: elcarose@uol.com.br

3. Participante do projeto, Departamento de Saúde, Universidade Estadual de Feira de Santana, e-mail: gsrocha12@yahoo.com

\section{PALAVRAS-CHAVE: Saúde Ocupacional; Estratégia de Saúde da Família; Sofrimento Psíquico.}

\section{INTRODUÇÃO}

O Programa Saúde da Família (PSF) foi criado em 1994 pelo Ministério da Saúde. Atualmente chamado Estratégia Saúde da Família (ESF), esse modelo de reorganização da atenção básica à saúde no Brasil, configurando-se como porta de entrada ao Sistema Único de Saúde (SUS) (BRASIL, 1997) As condições de trabalho em que as equipes estão inseridas variam, dentre outros fatores, incluem-se a situação político-administrativa e estrutural de cada município ou localidade, que determinam formas de contratação, infraestrutura, materiais, recursos humanos, dinâmica da assistência e condições políticas para desenvolvimento do trabalho, influenciando positiva ou negativamente no seu impacto, assim como na saúde dos trabalhadores.

Para Goldberg e Huxley (1993), a expressão Distúrbio Psíquico Menor (DPM) foi criada para designar sintomas de insônia, fadiga, irritabilidade, esquecimento, dificuldade de concentração e queixas somáticas que demonstram ruptura do funcionamento normal do indivíduo, mas não configuram categoria nosológica da $10^{\mathrm{a}}$ Classificação Internacional de Doenças (CID-10), nem dos Manuais de Diagnósticos e Estatísticas (DSM) da Associação Psiquiátrica Americana. Entretanto constituem problema de saúde pública em função das demandas geradas aos serviços de saúde e do absenteísmo no trabalho (COUTINHO; ALMEIDA-FILHO; MARI, 1999).

Segundo a Organização Mundial de Saúde (OMS, 2003), estima-se a ocorrência de $25 \%$ de DPM e 5 a $10 \%$ de transtornos mentais graves em indivíduos com idade igual ou superior a 18 anos, tendo como fatores de maior carga da doença: a pobreza, sexo, idade, doenças físicas, fatores familiares e ambientais (OMS, 2003)

\section{MATERIAL E MÉTODOS OU METODOLOGIA (ou equivalente)}

Trata-se de um estudo de revisão integrativa (RI) sobre a prevalência e fatores associados do DPM em trabalhadores que atuam na Estratégia de Saúde da Família e cumpriu a recomendação PRISMA para relato de revisões sistemáticas e meta- análises. Dois revisores trabalharam de forma independente, na condução do trabalho. (GALVÃO; PANSANI; HARRAD, 2015)

A RI utilizou artigos de bases eletrônicas nacionais e internacional de artigos foram identificados nas bases de dados Medline (National Library of Medicine, Bethesda, MD), Lilacs (Centro Latino-Americano e do Caribe de Informação em Ciências da Saúde - Bireme), Scielo (Scientific Electronic Library Online) e PubMed ( U.S. National Library of Medicine).

Foram considerados todos os artigos, dissertações e teses contendo a combinação dos descritores (em português e inglês), disponibilizados no site http://decs.bvs.br/. Os seguintes 
descritores foram utilizados: sofrimento psíquico/ stress, psychological; programa saúde da família/ family health program and saúde ocupacional/ occupational health.

Pelo BIREME, a fim de encontrar estudos nos bancos de dados Lilacs, Medline e Pubmed foram utilizados os descritores: Family health strategy, Occupational health e Psychic suffering. Na etapa inicial da busca 07 publicações científicas foram encontradas, sendo 02 duplicadas, restaram 05 publicações sendo uma excluída pelo título, na totalidade foram 03 publicações ( 01 artigos e 02 teses).

\section{RESULTADOS E/OU DISCUSSÃO (ou Análise e discussão dos resultados)}

\begin{tabular}{|c|c|c|c|c|}
\hline Autor (es) & $\begin{array}{c}\text { Ano da } \\
\text { Publicação }\end{array}$ & Modalidade & Título & Objetivos \\
\hline Carreiro & 2010 & Dissertação & $\begin{array}{l}\text { O impacto do trabalho na } \\
\text { saúde mental dos } \\
\text { profissionais da estratégia } \\
\text { de saúde da família }\end{array}$ & $\begin{array}{l}\text { Identificar entre os trabalhadores com risco } \\
\text { para depressão e ansiedade, identificados } \\
\text { em uma pesquisa anterior, aqueles que } \\
\text { desenvolveram algum tipo de adoecimento; } \\
\text { investigar entre os trabalhadores que } \\
\text { adoeceram os fatores predisponentes e } \\
\text { estratégias utilizadas para prevenção do } \\
\text { adoecimento mental. }\end{array}$ \\
\hline Ribeiro & 2006 & Dissertação & $\begin{array}{l}\text { O sofrimento psíquico dos } \\
\text { trabalhadores de uma } \\
\text { equipe do Programa Saúde } \\
\text { da Família na organização } \\
\text { do trabalho }\end{array}$ & $\begin{array}{l}\text { Identificar a relação entre o sofrimento } \\
\text { psíquico do trabalhador e a organização do } \\
\text { trabalho em uma equipe do PSF. }\end{array}$ \\
\hline Barbosa et al & 2012 & $\begin{array}{l}\text { Estudo } \\
\text { epidemiológic } \\
\text { o de corte } \\
\text { transversal }\end{array}$ & $\begin{array}{l}\text { Trabalho e saúde mental } \\
\text { dos profissionais da } \\
\text { Estratégia } \\
\text { Saúde da Família em um } \\
\text { município do Estado da } \\
\text { Bahia, Brasil }\end{array}$ & $\begin{array}{l}\text { Descrever características sócio } \\
\text { demográficas e do trabalho e a prevalência } \\
\text { de Distúrbios Psíquicos Menores (DPM) e } \\
\text { de positivos ao teste CAGE (abuso no } \\
\text { consumo de álcool) entre médicos, } \\
\text { enfermeiros e cirurgiões-dentistas da ESF } \\
\text { de Feira de Santana, Bahia. }\end{array}$ \\
\hline $\begin{array}{l}\text { Katsurayama } \\
\text { et al }\end{array}$ & 2013 & Estudo teórico & $\begin{array}{l}\text { Trabalho e sofrimento } \\
\text { psíquico na } \\
\text { Estratégia Saúde da } \\
\text { Família: } \\
\text { uma perspectiva Dejouriana }\end{array}$ & $\begin{array}{l}\text { Análise teórica do constructo "sofrimento } \\
\text { psíquico" do trabalhador na Estratégia } \\
\text { Saúde da } \\
\text { Família (ESF) }\end{array}$ \\
\hline
\end{tabular}

No Quadro 01 foram apresentados os 02 artigos e 02 teses selecionadas. Na análise dos artigos Katsurayama (2013) apresenta contribuições teóricas sobre o tema "sofrimento psíquico" do trabalhador na Estratégia Saúde da Família (ESF), apontando perspectivas de enfrentamento desse problema de saúde mental com estratégias de acompanhamento dos profissionais com escuta e o redirecionamento das políticas públicas de atenção básica voltada para os trabalhadores de saúde e funcionários do SUS. O segundo artigo, de recorte epidemiológico Barbosa e colaboradores (2012) trata-se de uma pesquisa de campo com profissionais da ESF com rastreamento pelo Self Reporting Questionnaire (SRQ-20) em médicos, enfermeiros e dentistas no município de Feira de Santana- Ba.

Carreiro (2010) nessa revisão integrativa apresentou os resultados de uma pesquisa proveniente de sua dissertação com abordagem qualitativa, onde foi utilizada a observação participante e entrevista não estruturada para o levantamento dos dados sobre o sofrimento psíquico em médicos, enfermeiros, dentistas, técnicos de enfermagem, Auxiliares de Consultório Dentário- ACD e Agentes comunitários de Saúde- ACS da ESF. Por fim a tese de Ribeiro (2006) teve resultados de uma pesquisa com caráter qualitativo e enfoque da epidemiologia social com a análise de entrevistas de médicos, enfermeiros e Agentes 
Comunitários de Saúde que deram origem a seis classes/categorias temáticas. Quanto ao local os estudos foram realizados em São Paulo, Paraíba e Bahia.

Os principais achados no estudo de Carreiro (2010) foram a descrição do perfil da população do estudo com características sócio demográficas mais prevalentes em ambos os grupos: sexo feminino, estado civil casado, religião católica, tempo de trabalho na ESF acima de 5 anos, ensino superior completo, sendo que a escolaridade ensino médio teve representação igual no grupo que teve algum adoecimento, que recebem entre 1 e 5 salários mínimos e desempenham a função de ACS, o que pode ser explicado pelo fato de a maior parte da amostra constituir-se por trabalhadores com essas características. Dos trabalhadores em risco de adoecimento, 61,1\% referiram que adoeceram, enquanto que 38,9\% não adoeceram.

Barbosa e colaboradores (2012) corroboram com outros estudos de Nascimento Sobrinho et al. (2006), Barros et al. (2008) e de Moroni e Paz (2011) ao encontrar resultados onde $76,3 \%$ dos sujeitos foram do sexo feminino. Com relação à idade, a média encontrada foi de $38,4 \pm 13,3$. Entre os médicos, uma maior frequência do sexo masculino $(54,2 \%)$ e faixa etária maior que 38 anos $(56,2 \%)$ mostrou- se mais prevalente. Foram encontrados nesse perfil que metade dos médicos que atuam na ESF de Feira de Santana apresenta idade entre 25 e 30 anos e entre 60 e 78 anos (19,1\% e 27,7\% respectivamente). Tais resultados caracterizam uma polarização indicando que a medicina na ESF está sendo exercida por profissionais jovens ou da terceira idade.

Na pesquisa de Barbosa e colaboradores (2012) outras categorias foram estudadas como enfermeiros e cirurgiões-dentistas, predominou o sexo feminino, 95,8\% e 78,8\% respectivamente, e a faixa etária menor ou igual a 38 anos, 77,5\% e 57,6\% respectivamente. Quanto à situação conjugal, nas três categorias foi mais frequente a de casados $(51,3 \%)$. Quanto à carga horária semanal geral (ESF e outros vínculos) de trabalho, 55,3\% dos médicos e $51,5 \%$ cirurgiões-dentistas ultrapassaram 40 horas semanais de trabalho, enquanto que 96,9\% dos enfermeiros apresentam carga horária de trabalho menor ou igual a 40 horas. Estes resultados são semelhantes aos resultados dos estudos de (Araújo, Graça e Araújo, 2003, Kirchof et al. 2009, Sousa 2010; Moroni e Paz, 2011).

A prevalência de DPM entre as categorias estudadas foi de $16,0 \%$, sendo entre os médicos de $17,4 \%$ entre os enfermeiros de $15,5 \%$ e entre os cirurgiões-dentistas de $15,2 \%$.

Ribeiro (2006) mostrou que o sofrimento psíquico do trabalhador na organização do trabalho na ESF foi relatado em momentos diversos desses estudos, desde experiências agudas e pontuais até um processo contínuo, vivenciado diariamente.

O trabalho na ESF foi relatado pelos profissionais como o principal causador de sofrimento psíquico, devido a problemas tanto de infraestrutura, como local inapropriado para atendimento, ambientes abafados, bem como organização do trabalho: aumento de cobrança administrativa do gestor, falta de funcionários, sobrecarregando os outros, desenvolvimento de múltiplas atividades, dificuldades de relacionamento entre os membros da equipe.

\section{CONSIDERAÇÕES FINAIS (ou Conclusão)}

A atual conjuntura da sociedade capitalista intensifica o trabalho, exigindo cada vez mais do trabalhador um enorme consumo da capacidade física e psicológica frente à quantidade de fatores de risco a que se expõe. No entanto, a ausência de trabalho também pode levar o trabalhador ao adoecimento, tanto quanto os ambientes laborais inadequados, a forma de organização do trabalho, a falta de valorização do desempenho e a participação insatisfatória nas decisões.

De certa maneira, o trabalhador está inserido contraditoriamente num processo de reestruturação da atenção a saúde, a Estratégia de Saúde da Família (ESF), mas ao mesmo 
tempo, amarrado por uma lógica ainda taylorista, capitalista e neoliberal que preza pela quantidade levando a quadros de desgaste e exaustão.

As lacunas de estudos e a necessidade de novas pesquisas devem ser recomendadas para o tema no sentido de conhecer o perfil e fatores associados do adoecimento mental, assim como pesquisas com abordagens qualitativas sobre os sentimentos e experiências vivenciadas pelos trabalhadores de saúde na ESF.

\section{REFERÊNCIAS}

1. BRASIL. Ministério da Saúde. Saúde da família: uma estratégia para a reorientação do modelo assistencial. Brasília, DF, 1997.

2. GOLDBERG, D.; HUXLEY, P. Common mental disorders: a bio-social model. 2 ed. London: Tavistock/Routledge: 1993.

3. COUTINHO, E. S. F.; ALMEIDA-FILHO, N.; MARI, J. Fatores de risco para morbidade psiquiátrica menor: resultado de um estudo transversal em três áreas urbanas do Brasil. Revista de Psiquiatria Clínica, v. 26, n. 5, p. 246-256, 1999

4. GALVÃO TF, PANSANI TSA, HARRAD D, tradutores. Principais itens para relatar Revisões sistemáticas e Meta-análises: A recomendação PRISMA. Epidemiol Serv Saude. 2015 abr-jun;24(2):335-42.

5. KATSURAYAMA, M; PARENTE, RCP; MORAES, RD; MORETTI-PIRES, RO. Trabalho e sofrimento psíquico na Estratégia Saúde da Família: uma perspectiva Dejouriana. Caderno de Saúde Coletiva, Rio de Janeiro, v. 21 (4), 414-9, 2013.

6. RIBREIRO, SFR. O sofrimento psíquico dos trabalhadores de uma equipe do Programa Saúde da Família na organização do trabalho. 2006. 162f. Dissertação ( Mestrado em Saúde Coletiva)- Universidade Estadual Paulista. Botucatu- São Paulo. 2006.

7. CARREIRO, GSP. O IMPACTO DO TRABALHO NA SAÚDE MENTAL DOS PROSISSIONAIS DA ESTRATÉGIA DA SAÚDE DA FAMÍLIA. 2010. 99f. Dissertação (Mestrado em enfermagem)- Centro de Ciencias da Saúde. Universidade Federal da Paraíba. João Pessoa- Paraíba. 2010

8. BARBOSA, GB; CORREIA, AKS; OLIVEIRA, LMM; SANTOS, VC; FERREIRA, SMS; JÚNIOR, DFM; SOBRINHO, CLN. Trabalho e saúde mental dos profissionais da Estratégia

9. NASCIMENTO SOBRINHO, C. L. et al. Condições de trabalho e saúde mental dos médicos de Salvador, Bahia, Brasil. Cadernos de Saúde Pública, Rio de Janeiro, v. 22, n. 1, p. 131-140, jun. 2006.

10. BARROS, D. S. et al. Médicos plantonistas de unidade de terapia intensiva: perfil sócio-demográfico, condições de trabalho e fatores associados à síndrome de burnout. Revista Brasileira de Terapia Intensiva, v. 20, n. 3, p. 235-240, 2008.

11. MORONI, G.; PAZ, A. A. Transtornos mentais menores em trabalhadores da Estratégia Saúde da Família na Serra Gaúcha. In: SEMINÁRIO INTERNACIONAL SOBRE O TRABALHO NA ENFERMAGEM, 3., 2011, Bento Gonçalves, RS. Anais... Bento Gonçalves: Associação Brasileira de Enfermagem, 2011. Disponível em: http://www.abeneventos.com.br/3siten/siten-trabalhos/files/0103.pdf>. Acesso em: 30 Jun. 2017 